\title{
Modeling particulate self-healing materials and application to uni-axial compression
}

\author{
Olaf Herbst · Stefan Luding
}

Received: 9 June 2008 / Accepted: 2 December 2008

(C) The Author(s) 2009. This article is published with open access at Springerlink.com

\begin{abstract}
Using an advanced history dependent contact model for DEM simulations, including elastoplasticity, viscosity, adhesion, and friction, pressuresintered tablets are formed from primary particles. These tablets are subjected to unconfined uni-axial compression until and beyond failure. For fast and slow deformation we observe ductile-like and brittle softening, respectively. We propose a model for local selfhealing that allows damage to heal during loading such that the material strength of the sample increases and failure/softening is delayed to larger strains. Local healing is achieved by increasing the (attractive) contact adhesion forces for those particles involved in a potentially breaking contact. We examine the dependence of the strength of the material on (a) the damage detection sensitivity, (b) the damage detection rate, and (c) the (increased) adhesion between healed contacts. The material strength is enhanced, i.e., the material fails at larger strains and reaches larger maximal stress values, when any of the parameters (a)-(c) is increased. For very large adhesion between the healed contacts an interesting instability with strong (brittle) fluctuations of the healed material's strength is observed.
\end{abstract}

O. Herbst $\cdot$ S. Luding $(\varangle)$

Multi Scale Mechanics, TS, CTW, UTwente, P.O. Box 217, 7500 AE Enschede, The Netherlands

e-mail: s.luding@utwente.nl

\section{O. Herbst}

Aerospace Engineering, TU Delft, Kluyverweg 1, 2629 HS Delft, The Netherlands

e-mail: olaf.herbst@gmx.net
Keywords Self-healing materials · Granular materials $\cdot$ Particle simulation $\cdot$ Contact force-laws · Friction · Adhesion . Elasto-plastic contact deformation

PACS 45.70. $-\mathrm{n} \cdot 46.50 .+\mathrm{a} \cdot 61.43 . \mathrm{Gt}$. 81.05.QK $\cdot 81.05 . \mathrm{Rm}$

\section{Introduction}

Self-healing materials encompass a wide range of materials capable of restoring their functionality. They are inspired by healing mechanisms in biological systems (Trask etal. 2007a). For example, the first healing of skin (or other tissues) results from coagulating blood. Ideally, self-healing takes place locally at the damaged site and does not require an (additional) external trigger. Applications of self-healing materials include the extension of service life of infrastructure or machinery (Dry 1996a). In the future, advanced self-healing capabilities may be crucial for the design and safety of new light-weight airplanes and space applications (Williams et al. 2007; Kessler 2007).

Efforts to design such novel engineering materials able to heal cracks autonomously have picked up considerably in recent years ( $\mathrm{Li}$ et al. 1998; White et al. 2001; Cordier et al. 2008). Intrinsic, self-activated healing has been described in cement as early as 1918 (Abrams 1918) and a number of cementitious healing mechanisms, both temporary and long lasting, have 
since been described, see, e.g., Refs. Hearn (1998); Dry (1994). In recent years self healing properties have been incorporated into other materials as well (van der Zwaag 2007), often involving polymers. Functionality restoration has been incorporated for crack healing inside the matrix of composite materials (White et al. 2001; Dry 1996a; Dry; White etal.; Brown etal. 2003a, b, 2005; Trask et al. 2007b; Kersey et al. 2007; Williams et al. 2007) but also for, e.g., nano-coatings (Feng et al. 2007; Shchukin and Möhwald 2007). Advances have been achieved with respect to designing a number of self-healing metal alloys, where diffusion is responsible for self-healing, as well as for semiconductors (van der Zwaag 2007).

In polymers self healing is usually achieved by the inclusion of a healing agent in either microcapsules (see, e.g., Ref. White et al. (2001), using a monomer), or hollow glass fibres (Trask et al. 2007b; Williams et al. 2007) filled with epoxy resin, and a catalyst, either spread out throughout the material or in hollow glass fibres. When the material rips, microcapsules or rods can break and release the healing agent. When the healing agent comes into contact with the catalyst, it solidifies (in many cases through polymerization) restoring (part of) the strength of the material. Polymers can achieve up to $100 \%$ of fracture strength after healing, depending on the type of polymer, and the choice of self-healing agents (Brown etal. 2003b). Unfortunately, the introduction of a healing agent and catalyst into the material may compromise the initial strength of the material. Other healing mechanisms include the usage of non-covalent hydrogen bonds to control the polymer network (Sijbesma etal. 1997). While some of the healing mechanisms require (exogeneous) heating (Trask et al. 2007b), others are capable of healing at ambient temperatures (White etal. 2001; Brown et al. 2003a, b, 2005; Kessler etal. 2003; Mauldin et al. 2007).

Efforts in designing self-healing cements have started much earlier (Soroker and Denson 1926; Brandeis 1937; Turner; Wagner 1974). In addition to methods similar to the ones used in polymers, i.e., using hollow fibres containing superglue (Li et al. 1998), many healing mechanisms are water-based (Hearn 1998). These can be categorized into two groups: autogeneous healing and hydration. The first group describes healing mechanisms where the restored functionality persists in dry condition while the second group describes systems that must remain saturated with water.
Computer based modeling of self-healing materials is not yet established. For solid materials, most approaches can be categorized as either continuum approaches, e.g., continuum damage models (CDM), or discrete element methods (DEM). The atomistic molecular dynamics methods that have been used for, e.g., modeling crack growth (White etal. 2004) shall only be mentioned here and not discussed further, since they typically describe much smaller length-scales than both DEM or continuum methods.

While continuum approaches are extremely successful within their limits they require empirical constitutive relations for the material. They act on a coarse grained level and the material must be (or is assumed to be) sufficiently homogeneous (or "slowly changing") on that coarse grained level. Continuum approaches therefore can miss important details on smaller scales. For self healing materials almost all theoretical work is based on continuum approaches for different types of materials, see, e.g., Refs. Barbero etal. (2005); Peizhen etal. (2000); Balazs (2007). More specific examples for continuum models include the description of a material with nanoporous glass fibres containing glue (Priman etal. 2007), a memory alloy composite (Burton et al. 2006) or nanoscale copper and biomaterial clusters (Guo and Guo 2006). Furthermore, a continuum model approach for a self healing material with enclosed capsules with glue as presented in 2001 by White and collaborators (White etal. 2001) has been published (Maiti et al. 2006).

One way to simulate a material by means of particle simulations is to sinter a sample, e.g., a tablet, from primary particles to create a dense granular packing. Granular materials are a very active field of research (Jaeger et al. 1989, 1990; Jaeger and Nagel 1992; Behringer 1993; Goldhirsch and Zanetti 1993; Behringer and Baxter 1994; Luding et al. 1994a; Sela and Goldhirsch 1998; Herbst et al. 2000, 2005; Santos 2008) and a natural toy model for material science in general and selfhealing materials in particular. Cohesive, frictional, fine powders show a peculiar flow behavior (Tomas 2004; Castellanos 2005; Luding 2005a,b). Adhesionless powder flows freely, but when adhesion due to van der Waals forces is strong enough, agglomerates or clumps can form and break into pieces again (Thornton and Yin 1991; Thornton etal. 1996; Kafui and Thornton 2000; Thornton and Antony 2000). This is enhanced by pressure- or temperature-sintering (Luding et al. 2005) and, under extremely strong pressure, tablets or 
granulates can be formed (Luding 2008; Luding and Suiker 2008) from the primary particles.

Many-particle simulations like the discrete element model (DEM) ( Cundall and Strack 1979; Bashir and Goddard 1991; Herrmann et al. 1998; Thornton 2000; Thornton and Zhang 2001; Vermeer et al. 2001) complement experiments on the scale of small "representative volume elements" (RVEs). They allow deep and detailed insight into the kinematics and dynamics of the samples since all information about all particles and contacts is available at all times. DEM requires only the contact forces and torques as the basic input to solve the equations of motion for all particles in such systems. Furthermore, the macroscopic material properties, such as, among others, elastic moduli, cohesion, friction, yield strength, dilatancy, or anisotropy can be measured from such RVE tests.

Research challenges involve not only realistic DEM simulations of many-particle systems and their experimental validation, but also the transition from the microscopic contact properties to the macroscopic behavior (Luding 2005a, b; Vermeer et al. 2001, 2004; Agnolin et al. 2006). This so-called micro-macro transition (Luding 2005a, b) should allow to better understand the collective flow behavior of many particles as a function of their contact properties.

In a self healing model presented recently (Luding and Suiker 2008) the adhesion between all particles is increased instantaneously at a given time (or strain) to simulate the healing ("global healing"). While this may be a reasonable model for, e.g., healing through temperature induced sintering it is not a very realistic model for those kinds of self healing materials, where, e.g., the breakage of microcapsules causes cracks to heal locally. For this reason we present a simple model where healing is activated locally where and when (potential) damage is detected. We examine the effect of the model parameters (a) damage-detection sensitivity, (b) damage-detection rate, and (c) healing adhesion.

The paper is organized as follows. After introducing the simulation method in Sect.2, the preparation of our samples is discussed in Sect.3. In Sect. 4 we introduce our self-healing model. In Sect. 5 we discuss a self-healing material under compression. Summary and conclusions are given in Sect. 6 together with a discussion of the relevance of our model for "real" experiments and materials.

\section{Discrete particle model}

To simulate packing, failure under compression, and self-healing in a granular material we use a Discrete Element Model (DEM) (Luding 2008; Cundall and Strack 1979; Bashir and Goddard 1991; Herrmann et al. 1998; Thornton 2000; Thornton and Zhang 2001; Lätzel et al. 2003). In the following we briefly introduce the method that allows us to simulate self-healing solid materials as granular packings. The numerics and algorithms are described in text books (Allen and Tildesley 1987; Rapaport 1995; Pöschel and Schwager 2005), so we only discuss the basic input into DEM, i.e., the contact force models and parameters, see Ref. Luding (2008) and references therein. We will, however, discuss in more detail the new self-healing model based on the existing model.

Inter-particle forces typically are assumed to depend pairwise on the overlap and the relative motion of two particles. This might not be sufficient to account for the inhomogeneous stress distribution inside the particles and possible multi-contact effects. However, this simplifying assumption makes it possible to study larger samples of particles with a minimal complexity of the contact properties while taking into account important phenomena like non-linear contact elasticity, plastic deformation, and adhesion as well as friction.

\subsection{Contact force laws}

Realistic modeling of the deformations of only two particles in contact with each other is quite challenging by itself. The description of many-body systems where each particle can have multiple contacts is extremely complex. We therefore, assume our particles to be nondeformable perfect spheres which interact only when in contact. We call two particles in contact when the distance of their centers of mass is less than the sum of their radii. For two spherical particles $i$ and $j$ in contact, with radii $a_{i}$ and $a_{j}$, respectively, we define their overlap

$\delta=\left(a_{i}+a_{j}\right)-\left(\boldsymbol{r}_{i}-\boldsymbol{r}_{j}\right) \cdot \boldsymbol{n}>0$

with the unit vector $\boldsymbol{n}:=\boldsymbol{n}_{i j}:=\left(\boldsymbol{r}_{i}-\boldsymbol{r}_{j}\right) /\left|\boldsymbol{r}_{i}-\boldsymbol{r}_{j}\right|$ pointing from $j$ to $i$. $\boldsymbol{r}_{i}$ and $\boldsymbol{r}_{j}$ denote the position of particle $i$ and $j$, respectively.

The force $\boldsymbol{f}$ on particle $i$, labeled $\boldsymbol{f}_{i}$, is modeled to depend pairwise on all particles with which particle $i$ 
is in contact, $\boldsymbol{f}_{i}=\sum_{j} \boldsymbol{f}_{i \mid j}^{c}$, where the sum runs over all particles in contact with particle $i$ and $f_{i \mid j}^{c}$ is the force on particle $i$ exerted by particle $j$ at contact $c$. The force $\boldsymbol{f}_{i \mid j}^{c}$ can be decomposed into a normal and a tangential part, $\boldsymbol{f}_{i \mid j}^{c}=f_{i \mid j}^{n} \boldsymbol{n}+f_{i \mid j}^{t} \boldsymbol{t}$, where $\boldsymbol{n} \cdot \boldsymbol{t}=0$. We will leave out the index $i \mid j$ from now on. In the following, we will first discuss the normal part of the force and then the tangential part.

\subsection{Normal contact forces}

To model the normal component $f^{n}=f_{e l}^{n}+f_{v}^{n}$ of the force we use an adhesive, elasto-plastic contact law that depends on three variables only and is described in more detail in Ref. Luding (2008): In this model the force between two spheres depends only on their overlap $\delta$, the relative velocity of their surfaces (including the relative normal and tangential velocity, i.e., it depends on the translational and rotational velocities of the two particles), and the maximum overlap $\delta_{\max }$ this contact has suffered in the past.

More specifically, we apply (a modification of) one of the simplest elasto-plastic models: a modified springdashpot model. The dashpot is, as usual, a viscous damping force that depends linearly on the normal component of the relative velocity (i.e., $f_{v}^{n}=\gamma_{n} \dot{\delta}$ ). The force associated with the spring depends linearly on the overlap $\delta$ (i.e., $f_{e l}^{n}=k_{*} \delta$ ) where, however, the stiffness "constant" $k_{*}$, itself depends on the history of the contact, see Fig. 1. The repulsive force during initial loading is governed by the initial loading stiffness constant $k_{1}$. The force $f_{*}^{n}=k_{*}\left(\delta-\delta_{*}\right)$ during un- and re-loading is proportional to an interpolated stiffness $k_{*}$, with $0<k_{1}<k_{*} \leq k_{2}$, and can be either repulsive or attractive. The history parameter $\delta_{\max }$ keeps in memory the maximal overlap previously reached. The maximal elastic stiffness constant, $k_{2}$, is used if the maximal overlap is greater than $\delta_{\text {material }}$. During unloading on the adhesive force branch, $f^{n}=-k_{c} \delta$, the history parameter $\delta_{\max }$ is continuously reduced. If a contact breaks $\delta_{\max }$ is set to zero.

The maximum allowed plastic overlap, $\delta_{\text {material }}=$ $k_{2} \delta_{\mathrm{f}} /\left(k_{2}-k_{1}\right)$, depends on the dimensionless model constant $\phi_{f}$ (typically $\phi_{f} \approx 0.05$ is used) which determines the "fluid-overlap" $\delta_{\mathrm{f}}=2 \phi_{f} a_{i} a_{j} /\left(a_{i}+a_{j}\right)$, for details see Ref. Luding et al. (2005). For overlaps larger than $\delta_{\text {material }}$, the force becomes linear with maximal stiffness $k_{2}$ (for convenience, for stability, and also

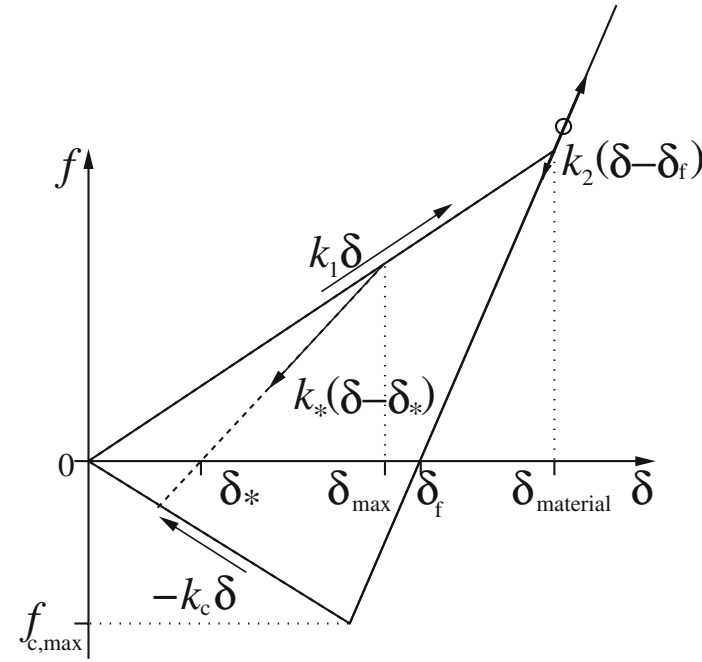

Fig. 1 Elasto-plastic part of the normal component of the contact force $f^{n}$, i.e., the part that is a (piece-wise linear) function of the overlap $\delta$. (To calculate the total normal force additionally the dashpot part has to be taken into account.) $k_{1}, k_{2}$, and $k_{c}$ are material stiffness constants and the $\delta$ with different sub-scripts denote special overlaps. For an explanation of the subscripts see the main text.

since such large deformations are not well represented by a pair-contact model).

Given all model parameter constants, $k_{1}, k_{2}, k_{c}$, and $\phi_{f}$, the maximum attractive force is

$f_{\mathrm{c}, \max }\left(k_{c}\right)=-\delta_{\mathrm{f}} \frac{k_{c} k_{2}}{k_{c}+k_{2}}$,

which leads to the maximally possible attractive force $f_{\mathrm{c}, \max }\left(k_{c} \gg k_{2}\right) \rightarrow-k_{2} \delta_{\mathrm{f}}$. Therefore, the contact adhesion saturates for large $k_{c} \gg k_{2}$. For very large $k_{c}$ we get an unstable (almost vertical) adhesive branch in the force model: any contact reaching this unstable branch immediately fails.

The motivation behind this model is to use the simplest model possible that is (at least piecewise) linear (in order to allow some theoretical analysis) while keeping the necessary phenomena such as plastic deformation, adhesion, and history dependence. For example, when two particles have been plastically deformed during loading their repulsive forces during unloading will be smaller than they were during loading and the contact will be completely unloaded before it reaches zero overlap (measured in terms of their original shape). When a real material is deformed it will memorize every deformation and the corresponding path. This is an extremely complex process. We simplify this 
complex process by keeping in memory only the maximum overlap a contact has suffered in the past. This allows us to model elasticity, plastic deformation, and adhesion to some extent. It does not represent all other possible complex mechanisms at contacts of particles. In that respect our model is a compromise between "as simple as possible" and "realistic enough" for our purposes.

\subsection{Tangential contact forces}

In the tangential direction, the forces and torques depend on the tangential displacement and the relative rotations of the particle surfaces. Dynamic (sliding) and static friction depend on the tangential component of the relative velocity of the contact points,

$\boldsymbol{v}_{t}=\boldsymbol{v}_{i j}-\boldsymbol{n}\left(\boldsymbol{n} \cdot \boldsymbol{v}_{i j}\right)$

where $\boldsymbol{v}_{i j}=\boldsymbol{v}_{i}-\boldsymbol{v}_{j}+a_{i}^{\prime} \boldsymbol{n} \times \boldsymbol{\omega}_{i}+a_{j}^{\prime} \boldsymbol{n} \times \boldsymbol{\omega}_{j}$

is the relative velocity of the particle surfaces at contact. Here, $a_{\alpha}^{\prime}=a_{\alpha}-\delta / 2$, for $\alpha=i, j$, is the corrected radius relative to the contact point. $\boldsymbol{v}_{i}, \boldsymbol{v}_{j}, \boldsymbol{\omega}_{i}$, and $\boldsymbol{\omega}_{j}$ are the linear and rotational velocities of particles $i$ and $j$, respectively.

Tangential forces $f^{t}$ acting on the contacts are modeled to be proportional to the accumulated sliding distance of the contact points along each other with a (tangential) stiffness constant $k_{t}$, i.e., $f^{t}=k_{t} \int v_{t} d t$. Including also a viscous dependent damping constant, $\gamma_{t}$, the tangential force is limited by the product of the normal force and the contact friction coefficient $\mu$, according to Coulombs law, $f^{t} \leq \mu f^{n}$, for more details see Ref. Luding (2008). Note, however, that this commonly used tangential force model is rather simple-leaving most of the complexity in the normal force model.

\subsection{Background viscous damping}

Viscous dissipation as mentioned above takes place localized in a two-particle contact only. In the bulk material, where many particles are in contact with each other, this dissipation mode is very inefficient for long-wavelength cooperative modes of motion, especially when linear force laws are involved Luding et al. (1994b). Therefore, an additional (artificial) damping with the background is introduced, such that the total force $\boldsymbol{f}_{i}$ and torque $\boldsymbol{q}_{i \mid j}:=a_{j}^{\prime} \boldsymbol{n} \times \boldsymbol{f}_{i \mid j}$ on particle $i$ are given by

$$
\begin{aligned}
\boldsymbol{f}_{i} & =\sum_{j} f^{n} \boldsymbol{n}+f^{t} \boldsymbol{t}-\gamma_{b} \boldsymbol{v}_{i} \quad \text { and } \\
q_{i} & =\sum_{j} \boldsymbol{q}_{i \mid j}-\gamma_{b r} a_{i}^{2} \boldsymbol{\omega}_{i},
\end{aligned}
$$

where the sums take into account all contact partners $j$ of particle $i$, and $\gamma_{b}$ and $\gamma_{b r}$ are the (artificial) background damping viscosities assigned to the translational and rotational degrees of freedom, respectively. The viscosities can be seen as originating from a viscous inter-particle medium and enhance the damping in the spirit of a rapid relaxation and equilibration. Note that the effect of $\gamma_{b}$ and $\gamma_{b r}$ should be checked for each set of parameters: it should be small in order to rule out artificial over-damping.

\subsection{Contact model parameters and units}

In the following we measure length in units of $1 \mathrm{~mm}$, mass in $1 \mathrm{mg}$ and time in $1 \mu \mathrm{s}$. Note that only a few parameters have to be specified with dimensions, while the others are expressed as dimensionless ratios in Table 1.

A maximal stiffness constant of $k_{2}=5$, as used in our simulations, corresponds to a typical contact duration (half-period) $t_{c} \approx 6.5 \times 10^{-4}$ for a typical collision of a large and a small particle with $\gamma=0$. Accordingly, an integration time-step $t_{\mathrm{MD}}=5 \times 10^{-6}$ is used in order to allow for a "safe" integration of the equations of motion. Note that not only the normal "eigenfrequency", but also the eigen frequencies for the rotational degrees of freedom have to be considered as well as the viscous response times $t_{\gamma} \approx m / \gamma$. All of the physical time scales (inverse eigen frequencies) should be considerably larger than $t_{\mathrm{MD}}$, while the viscous response times should be even larger, such that $t_{\gamma}>t_{c}>t_{\mathrm{MD}}$. A more detailed discussion of all the effects due to the interplay between the model parameters and the related times is, however, far from the scope of this paper and can be found in Ref. Luding (2008).

\section{Tablet preparation and material failure test}

\subsection{Tablet preparation}

Having introduced the model and its parameters in the last section we describe now the experimental setup 
Table 1 Values of the microscopic material parameters used (third column), if not explicitly specified

\begin{tabular}{|c|c|c|c|c|}
\hline Property & Symbol & Value & Dimensional units & SI-units \\
\hline Time unit & $t_{u}$ & 1 & $1 \mu \mathrm{s}$ & $10^{-6} \mathrm{~s}$ \\
\hline Length unit & $x_{u}$ & 1 & $1 \mathrm{~mm}$ & $10^{-3} \mathrm{~m}$ \\
\hline Mass unit & $m_{u}$ & 1 & $1 \mathrm{mg}$ & $10^{-6} \mathrm{~kg}$ \\
\hline Average particle radius & $a_{0}$ & 0.005 & $5 \mu \mathrm{m}$ & $5 \cdot 10^{-6} \mathrm{~m}$ \\
\hline Material density & $\rho$ & 2 & $2 \mathrm{mg} / \mathrm{mm}^{3}$ & $2000 \mathrm{~kg} / \mathrm{m}^{3}$ \\
\hline Max. loading/unloading stiffness & $k_{2}$ & 5 & $5 \mathrm{mg} / \mu \mathrm{s}^{2}$ & $5 \cdot 10^{6} \mathrm{~kg} / \mathrm{s}^{2}$ \\
\hline Initial loading stiffness & $k_{1} / k_{2}$ & 0.5 & & \\
\hline Adhesion parameter & $k_{c} / k_{2}$ & 0.2 & & \\
\hline Tangential stiffness & $k_{t} / k_{2}$ & 0.2 & & \\
\hline Coulomb friction coefficient & $\mu$ & 1 & & \\
\hline Normal viscosity & $\gamma=\gamma_{n}$ & $5 \cdot 10^{-5}$ & $5 \cdot 10^{-5} \mathrm{mg} / \mu \mathrm{s}$ & $5 \cdot 10^{1} \mathrm{~kg} / \mathrm{s}$ \\
\hline Tangential viscosity & $\gamma_{t} / \gamma$ & 0.2 & & \\
\hline Background viscosity & $\gamma_{b} / \gamma$ & 4.0 & & \\
\hline Background viscous torque & $\gamma_{b r} / \gamma$ & 1.0 & & \\
\hline Fluid overlap & $\phi_{f}$ & 0.05 & & \\
\hline
\end{tabular}

The fourth column contains these values in the appropriate units, i.e., when the time-, length-, and mass-units are $\mu \mathrm{s}, \mathrm{mm}$, and mg, respectively. Column five contains the parameters in SI-units. Energy, force, acceleration, and stress have to be scaled with factors of $1,10^{3}, 10^{9}$, and $10^{9}$, respectively, for a transition from dimensionless to SI-units

and the basic steps of our simulations. First, a "tablet" (granule) is prepared from primary particles which behave according to the contact force laws described above. A four-step process is applied:

(1) creation of a loose initial sample

(2) pressure sintering by isotropic compression

(3) removal of the pressure

(4) relaxation

On the resulting "tablet", or material sample, or RVE, tests can be performed, e.g., controlled compression or tensile tests, both on the "plain" tablet as well as under self-healing conditions. Care has to be taken to perform first the preparation and later the tests in a symmetric way (see below) to avoid artifacts.

\subsubsection{Initial sample}

Before sintering the tablet the first step is to create a loose configuration of $N=1,728$ spherical particles with a Gaussian distribution of radii with average $a_{0}=0.005$. The tails of the distribution are cut-off at 0.003 and 0.0075 to ensure that all particles are comparable in size (David et al. 2005), i.e., neither too large nor too small particles are desired. For the samples presented in this paper, the half-width of the distribution is $w_{a}=\sqrt{\left\langle a^{2}\right\rangle-\langle a\rangle^{2}}=0.00072$. In addition, the initial velocities are drawn from a Gaussian distribution in each direction.

In the initial preparation stage the particles are arranged on a regular cubic lattice with wide spacing so that particles are not in contact-neither with each other nor with a wall. Then, with $\mu=0$ and $k_{c}=0$ (i.e., zero friction and zero adhesion), the system is compressed with a pressure of $p_{1}=0.5$ to create a loose initial isotropic packing with (after relaxation) a coordination number $\mathcal{C}=5.89$ and volume fraction, $v=\sum_{i} V\left(a_{i}\right) / V=0.607$, where $V\left(a_{i}\right)=(4 / 3) \pi a_{i}^{3}$ is the volume of one particle $i$.

\subsubsection{Pressure sintering}

The second step is pressure sintering: the system is compressed by keeping one wall in each spatial direction fixed while applying a constant pressure of $p_{s}=$ 10 to the other (three) walls. During this compression, the particles are frictional with a friction coefficient $\mu=1$, and have zero adhesion among each other, i.e., $k_{c}=0$. Four of the six walls are frictionless, $\mu^{\text {wall }}=0$, and cohesionless, $k_{c}^{\text {wall }}=0$. The remaining two (opposing) walls are already prepared for the tests 
to come: these two walls define the uni-axial direction and are strongly adhesive, with $k_{c}^{\text {wall }} / k_{2}=20$, such that the sample sticks to them, while all other walls can be easily removed in the third step described below. The wall adhesion has no visible effect here, since the sample is strongly confined. In contrast, friction has an effect, i.e., friction with the walls would hinder the pressure to be transferred completely to the opposite wall. Frictional walls carry part of the load-an effect that has been known since the early work of Janssen (1895); Sperl (2006).

In Fig. 2 the kinetic energy and the mean coordination number are shown as functions of time during compression (step 2): the kinetic energy first increases with the smoothly and slowly increasing wall-velocity (see below). Then it decreases due to the energy dissipation in the system. After the desired pressure is reached, it is kept constant until the kinetic energy has very well reached a tiny value, within fluctuations only due the numerical accuracy limit.

A rather high volume fraction, $v=0.6754$, is reached during the pressure sintering. The correspond- ing coordination number is $\mathcal{C} \approx 7.17$ in this state. After stress-relaxation down to a negligible residual stress (see below), these values will decrease considerably to $v \approx 0.627$ and $\mathcal{C} \approx 6.097$, but remain larger than after the initial preparation.

\subsubsection{Pressure release}

Using the pressure sintered sample, the third step is to remove the pressure from the walls. Before, we do so $k_{c}$ is set to its desired initial value, i.e., $k_{c} / k_{2}=0.2$.

The control pressure is smoothly released from the walls in a co-sinusoidal way. Starting from its sintering value, $p_{s}=10$, down to a residual value, $p_{0}$, that is five orders of magnitude lower, i.e., $p_{0} / p_{s}=10^{-5}$. The half period of the co-sinusoidal pressure release is $t_{0}=12.5$, see first marker (open circle) in Fig. 3, but relaxation is continued further until the kinetic energy is dissipated and reaches tiny values at much larger times. The small residual pressure keeps single particles from accidentally leaving the sample and also keeps the walls close to the sample. (This is important in order to not
Fig. 2 Kinetic energy $E_{\text {kin }}$ (left) and coordination number $\mathcal{C}$ (right) as functions of time $t$ during isotropic pressure sintering with final pressure $p_{s}=10$. Here the particle contacts are adhesionless, $k_{c}=0$, while the other parameters are given in Table 1
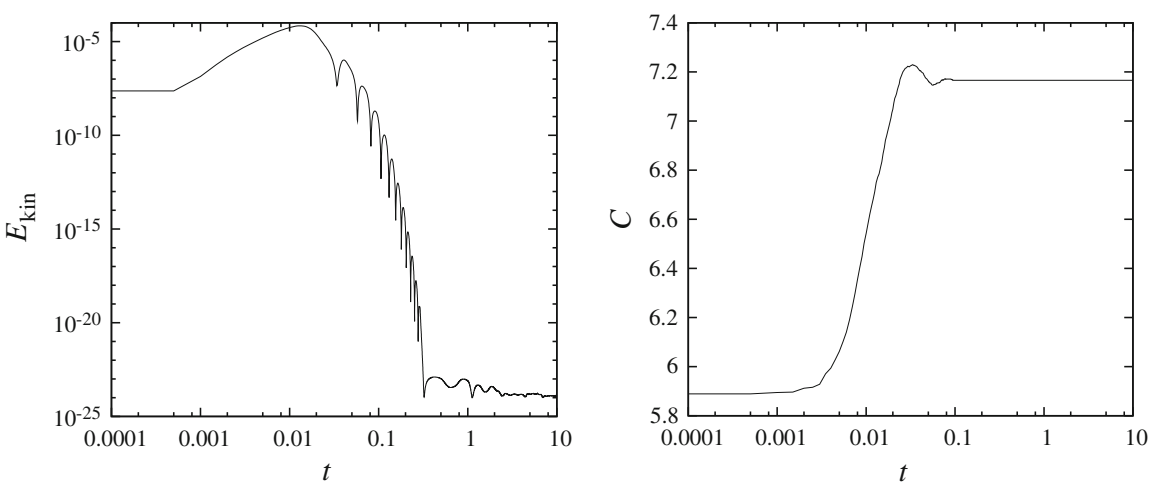

Fig. 3 Kinetic energy $E_{\text {kin }}$ (left) and coordination number $\mathcal{C}$ (right) as functions of time $t$ during smooth stress-removal from the walls and subsequent relaxation. The three markers in the left graph indicate the end of the pressure decrease and the two changes in the relaxation procedure described in the text
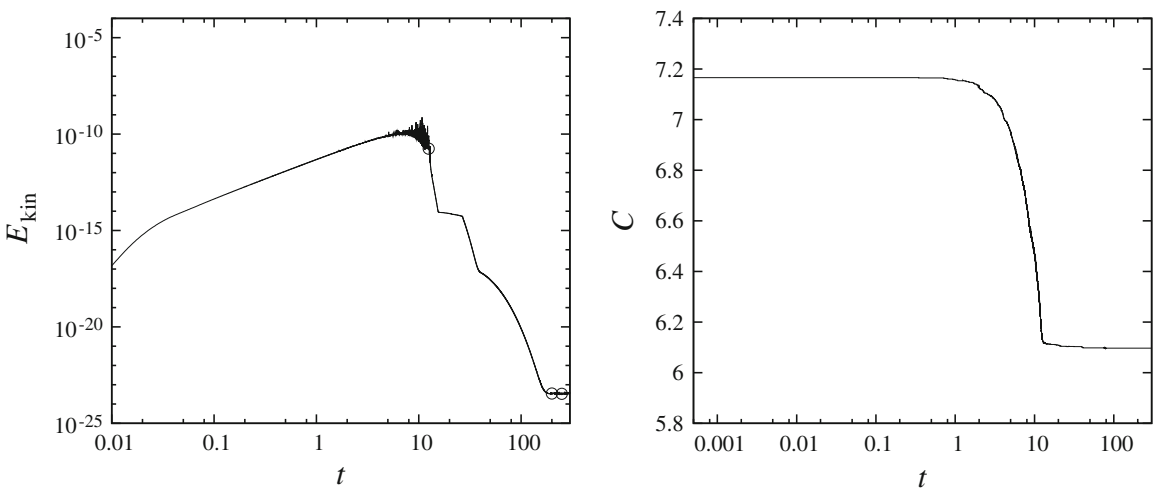
spoil the efficiency of our linked cell algorithm, where the cell size is a fraction of the system size between the walls.) However, this confining stress $p_{0} / p_{s}$ is not big enough to affect the dynamics of the tests performed, i.e., tests with $p_{0} / p_{s}=10^{-3}$ lead to practically identical results, concerning final density and coordination number.

\subsubsection{Final relaxation}

The fourth preparation step is the final relaxation of the system. This is done in three substeps. First, immediately after the control pressure on the wall has reached its low residual value, the system is relaxed further until time $t_{r_{1}}=200$ (with three fixed walls and three walls with the residual pressure reached at the end of the wall removal procedure in order to remove any residual center-of-mass velocity). Second, after the kinetic energy has reached and maintained the small value, within fluctuations, for a long time, the system is further relaxed and symmetrized with the same pressure of $p_{0}=10^{-4}$ applied from all sides for another time interval of $t_{r_{2}}=50$. In the final relaxation step, again a time interval of $t_{r_{3}}=50$, the two walls in the $x$-direction are fixed and the other four walls are kept at the residual pressure. We now have a representative volume element (RVE) sample with fixed walls in the $x$-direction and mobile walls with negligible stresses (i.e. virtually unconfined walls) in the $y$ and $z$ directions.

The prepared sample can now be used for all sorts of tests. It is almost cubical with side length $L_{0}=0.1133$. The tests to be described in this study are uni-axially deforming the walls in the $x$ direction while keeping the other walls at pressure $p_{0}=10^{-4}$. In the next subsection we will show a simple compression test of our particulate material without self-healing, before, in the rest of the paper, especially in Sect. 5, we will deal with compression tests of self-healing materials.

\subsection{Compression test}

In this section we describe an uni-axial compression test, starting from the final configuration as described in the previous subsection. This test resembles a direct measurement of the unconfined yield-strength, as applied in mechanical engineering and particle technology (Schwedes 2003).
We perform a strain controlled symmetric compression test by moving inwards two opposing walls. We directly move the walls in a cosinusoidal way for up to half a period, i.e., the relative wall position $\delta w(t)=$ $w(t)-w(0)=\left(A_{\cos } / 2\right)\left(1-\cos \left(2 \pi t / \tau_{\cos }\right)\right)$, with compression positive, where $A_{\cos }$ is the amplitude, or maximal compression distance, and $\tau_{\cos }$ is the compression "period". (Note that the maximal compression distance is reached after half a period.)

For a representative volume element (RVE) like our sample, we quantify the deformation in terms of the strain imposed from two sides $\varepsilon(t)=2 \delta w(t) / L_{0}$, with the maximum strain $\varepsilon_{\cos }=2 A_{\cos } / L_{0}$. The timederivative of the strain leads to the (time-dependent) compression rate $\mathcal{R}(t):=\partial \varepsilon / \partial t=\mathcal{R}_{\cos } \sin \left(2 \pi t / \tau_{\cos }\right)$ with the maximum compression rate $\mathcal{R}_{\cos }=2 \pi \varepsilon_{\cos } \tau_{\cos }^{-1}$ reached at a quarter-period.

Depending on the rate of compression different stress-strain behavior is observed, see related literature (Luding and Herrmann 2001). In Fig. 4 the stress-strain response for two different compression periods, $\tau_{\mathrm{cos}}=$ 8 (fast) and $\tau_{\mathrm{cos}}=320$ (quasi-static), is presented for an amplitude of $A_{\mathrm{cos}}=0.04$, i.e., $\varepsilon_{\mathrm{cos}} \approx 0.706$. The maximal compression rates $\mathcal{R}_{\mathrm{cos}}$ are reached at strain $\varepsilon_{\cos } / 2$ (data not shown). In this paper most compression tests are presented up to a maximal strain of $\varepsilon=0.05$, which is already in the critical flow regime. The peakstress, i.e., the maximal stress, is reached at smaller strains, $\varepsilon_{\max } \approx 0.01$, so that the relevant strain-rate is much smaller, but still proportional to the inverse period $1 / \tau_{\mathrm{cos}}$, which will be given in the following.

In the compression tests with small and moderate rates, the initial stress-strain relation is very close to linear, with slope $C=\partial \sigma / \partial \varepsilon=225.6$, i.e., the rate dependence is not visible in the elastic regime. Thus, up to a strain of about one percent the system behaves almost like an elastic solid, and only close to maximum stress and during softening, the rate effects become important.

Figure 5 shows the maximum stress as a function of the compression rate. Only the lowest data points $\left(1 / \tau_{\cos } \leq 0.01\right)$ are in the (truly) quasi-static regime: even slower compression rates lead to the same qualitative stress-strain diagrams - up to fluctuations. For larger rates the stress-strain behavior changes from quasi-brittle to ductile-like, i.e., from sharp drops to smoother behavior due to viscous damping. Therefore, we denote deformation rates $1 / \tau_{\cos } \leq 0.01$ as quasistatic, those with $0.01<1 / \tau_{\cos } \leq 0.2$ as (moderately) 
Fig. 4 Normalized axial stress plotted against axial strain for fast $\left(\tau_{\mathrm{cos}}=8\right.$ and $\left.1 / \tau_{\cos }=0.125\right)$ and slow

$\left(\tau_{\mathrm{cos}}=320\right.$ and

$\left.1 / \tau_{\mathrm{cos}}=0.003125\right)$

compression, with $\sigma_{0}=k_{2} / a_{0}=10^{3}$

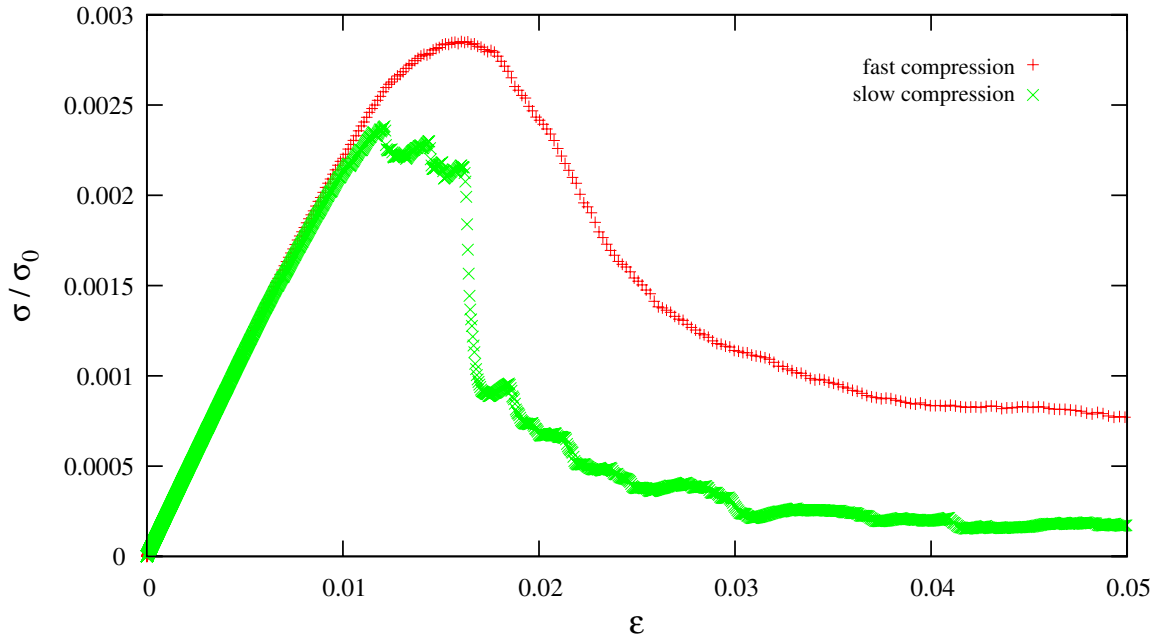

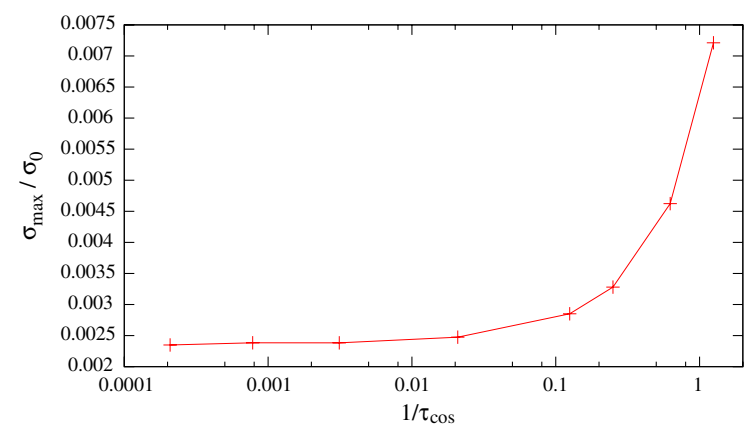

Fig. 5 Maximum (normalized) stress as a function of the maximum compression rate $1 / \tau_{\cos }$. Note the finite non-zero value as the compression rate becomes quasi-static. Note also that for fast compressions the increase in stress is significant, in the data presented in this graph up to three times the quasi-static value

fast, and those with $1 / \tau_{\cos }>0.2$ as very fast. The quasistatic compression is consistent with brittle material behavior, see, e.g., Fig. 3 in Ref. Brown and Sottos (2000).

\section{Self-healing model}

To model a self-healing material we need to locally detect damage and initiate local healing when damage is detected. Again we attempt to use a simple model to capture the essence of this complex process. Therefore, we introduce the following self-healing scheme that is compatible with (but not limited to) the contact model described in Sect. 2:

(1) detect damage (see below, Sect. 4.1),
(2) heal detected damage (see below, Sect. 4.2),

(3) run simulation for a time $\tau_{\mathrm{D}}$,

(4) go to 1.

This procedure allows us to set the damage detection rate $1 / \tau_{\mathrm{D}}$ at which damage is detected (and subsequent self-healing is activated), and to specify the healing sensitivity and the healing strength separately.

\subsection{Damage detection}

To detect damage we check each contact for two conditions:

(1) First, we probe the condition

$$
f^{n} \leq-\alpha k_{c} \delta \text {. }
$$

Here, $f^{n}$ is the normal component of the force between the two particles involved in the contact as introduced in Sect. 2.1 and $\alpha$ is a tuning constant to adjust the sensitivity of damage detection. A negative value of the force corresponds to an attractive force, see Figs. 1 and 6. (More details about the contact model can be found in Ref. Luding (2008).)

(2) Secondly, we require the particles to be moving apart from each other, ensuring that only those contacts are considered damaged which are still "endangered" of breaking immediately.

For moderate $\alpha<1$, the healing condition in Eq. 5 is fulfilled whenever a contact is close to breaking, see Fig. 6. In the extreme case of $\alpha \geq 1$, it is very unlikely that a contact fulfills this condition whereas, in the case of $\alpha \approx 0$ or $\alpha<0$ all contacts under weak tension or 


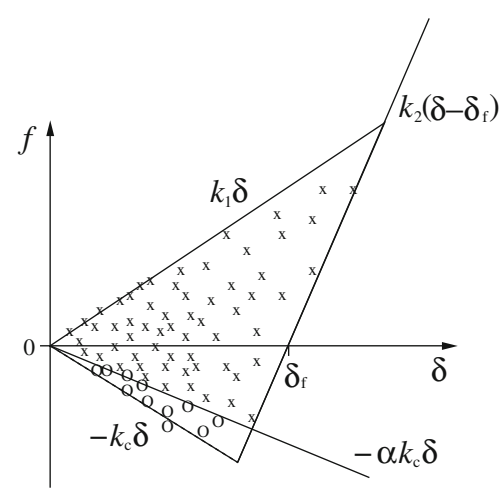

Fig. 6 Schematic plot of (some of) the normal forces typically present at a certain time in the system. Each elasto-plastic-adhesive contact is represented by a point inside the triangle. (Due to viscous forces some contacts may be situated outside of the triangle if the total normal force is considered.) Healing is applied if the force is smaller than $-\alpha k_{c} \delta$ (circles) and if the contact partners are separating

even under compression fulfill the condition of Eq. 5corresponding to over-sensitive healing.

This model is inspired by experiments, more precisely by experiments using a healing-agent confined to capsules which are spread out throughout the matrix material (White etal. 2001): When a certain force is excerted on a capsule it will rupture. Once a capsule is broken, the healing agent will flow out and solidify. At the same position, there cannot any further healing because there is no more healing agent available. Different types of capsules and capsule-matrix interface can be modeled by different healing sensitivities $\alpha$. The present model does not allow for massive volume changes (as due to foaming or bubbling healing agents).

Figure 7 shows the maximum stress reached during compression as a function of the sensitivity tuning parameter $\alpha$ (performing the healing described in Sect. 4.2 below). As expected, the maximum stress for a sample with self-healing and $\alpha \geq 1$ does not differ from the one reached without self-healing. For $\alpha<0$ the material sample becomes very strong since most contacts will be labeled "damaged" (and subsequently healed) at the very first detection step. For the rest of our study we will use $\alpha=0.9$. This value is in a regime where small changes in $\alpha$ will lead to a linear response in the maximum stress observed.

We have also tested applying only the force condition, i.e., initiating healing for all those contacts fulfilling the first condition, i.e., Eq. 5, regardless of their

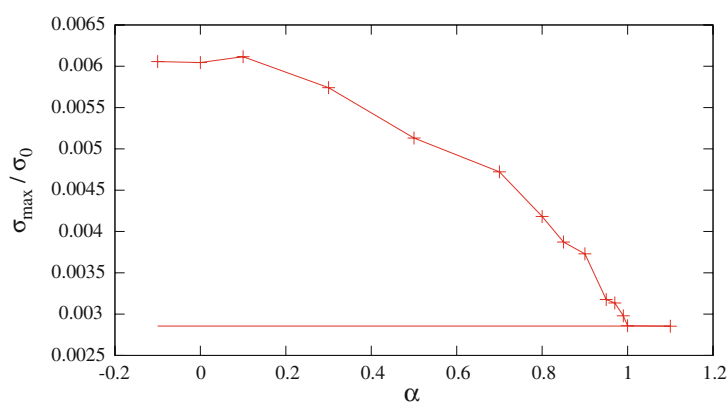

Fig. 7 Dependence of the maximum (normalized) stress reached by healing with different sensitivities $\alpha$, for the self-healing adhesion parameter $k_{\mathrm{SH}}=5$ and damage detection rate $1 / \tau_{\mathrm{D}}=$ 0.002, while otherwise the same parameters as for Fig. 4 (moderately fast) were used. The horizontal line shows the maximum strength for a sample without self healing

relative velocities. This leads to similar results with more contacts healed initially but otherwise similar behavior. Naturally, it leads to a small increase in the maximum stress (less than $10 \%$ for the cases studied). In the following only results will be presented where both healing criteria (force and separation) are active.

\subsection{Healing}

Where damage is detected, i.e., where the two damage conditions mentioned in Sect. 4.1 are met, we initiate self-healing which we model by (instantaneously) increasing $k_{c}$ to a higher value $k_{\mathrm{SH}}$ for both particles of that particular contact. This immediately increases their maximum possible attractive forces and thus delays or avoids the failure of their contact. Since the value of $k_{\mathrm{SH}}$ sets the final bonding strength of the healing agent, different healing agents can be modeled by using different values of $k_{\mathrm{SH}}$.

Note that all contacts parameters, e.g., $k_{c}$, are assigned to particles, not to contacts. This may (and usually will somewhere in the sample) lead to contacts whose two partners have different values of $k_{c}$. In that case we use an intermediate value $k_{c}^{\prime}=2 k_{c, 1} k_{c, 2} /\left(k_{c, 1}+\right.$ $k_{c, 2}$ ) (where $k_{c, 1}$ and $k_{c, 2}$ are the $k_{c}$ values for the two particles) for that contact. The reasoning behind this model is twofold: First it is much easier to keep track of the $k_{c}$ 's if they are connected to particles instead of contacts because this way one neither needs to keep track of past contacts after they have opened nor has to define new contact properties for a new contact. Secondly, it makes the healing a little more non-local 
(and less abrupt) like in the experimental situation where the self-healing agent spreads out a little from the point where the capsule broke.

\section{Compression tests of a self-healing sample}

In this section, compression tests of a self-healing material are presented. Using a fixed healing sensitivity $(\alpha=$ $0.9)$, the effects of the remaining two model parameters, i.e., the self-healing adhesion parameter, $k_{\mathrm{SH}}$, and the damage detection rate, $1 / \tau_{\mathrm{D}}$, will be studied in more detail.

\subsection{The extreme cases}

In Fig. 8, the stress-strain response of a reference sample without self-healing capability, $k_{c}=k_{\mathrm{SH}}=1$, is compared to different cases of self-healing. For all these cases, the compression is quasi-static, with compression period $\tau_{\cos }=320$. In order to considerably increase the adhesive force, a self-healing adhesion parameter $k_{\mathrm{SH}}>k_{c}$ is chosen. This corresponds to an increase of the maximum (possible) adhesive force by a factor of

$I_{\mathrm{SH}}:=\frac{f_{\mathrm{c}, \max }^{\mathrm{SH}}\left(k_{c}\right)}{f_{\mathrm{c}, \text { max }}\left(k_{c}\right)}=\frac{k_{\mathrm{SH}}\left(k_{c} / k_{2}+1\right)}{k_{c}\left(k_{\mathrm{SH}} / k_{2}+1\right)}$,

which we denote as the self-healing intensity in the following. In the case discussed here $\left(k_{c}=1, k_{\mathrm{SH}}=5\right.$, $\left.k_{2}=5\right), I_{\mathrm{SH}}=3$.

The two extreme cases are the reference sample with no healing at all $\left(k_{c}=k_{\mathrm{SH}}=1\right)$ and the case where
$k_{c}=k_{\mathrm{SH}}=5$ from the very beginning for all contacts. In both cases no damage detection is necessary. In the first case all contacts remain unhealed forever while in the latter case all contacts are already "healed" from the very beginning. This case corresponds to the "full" or "pre-emptive" or "global" healing mentioned in Ref. Luding and Suiker (2008). Not surprisingly, it leads to the strongest increase in material strength.

Additionally, two cases of local self-healing are shown. The only difference between them is their damage detection rate, all other parameters are the same as for the two extreme cases. The damage detection time intervals $\tau_{\mathrm{D}}=2$ and $\tau_{\mathrm{D}}=0.004$ are used, which correspond to slow $\left(1 / \tau_{\mathrm{D}}=1 / 2\right)$ and fast $\left(1 / \tau_{\mathrm{D}}=250\right)$ damage detection, respectively.

Even the slow damage detection increases the material strength (maximum stress sustained) by about $25 \%$. Fast damage detection leads to a considerably higher increase in strength (about 100\% compared to the sample without self-healing), but does not reach the extreme case of "full" or "pre-emptive" healing, which leads to an increase of about $150 \%$.

In the following, first, the influence of the damage detection rate is examined in more detail in Subsect. 5.2 , and second, the effect the healing intensity is studied in Subsect. 5.3.

\subsection{Variation of the damage detection rate}

In Fig. 9, the stress-strain response is studied for systems with different damage detection rates $1 / \tau_{\mathrm{D}}$ from 0.5 to 250 while all other parameters are kept constant.
Fig. 8 Stress-strain response of samples with (from top to bottom) "full pre-emptive" healing $\left(k_{c}=5\right)$, fast damage detection rate $\left(1 / \tau_{\mathrm{D}}=250\right)$, slow damage detection rate $\left(1 / \tau_{\mathrm{D}}=0.5\right)$, and without self-healing $\left(k_{c}=1\right)$

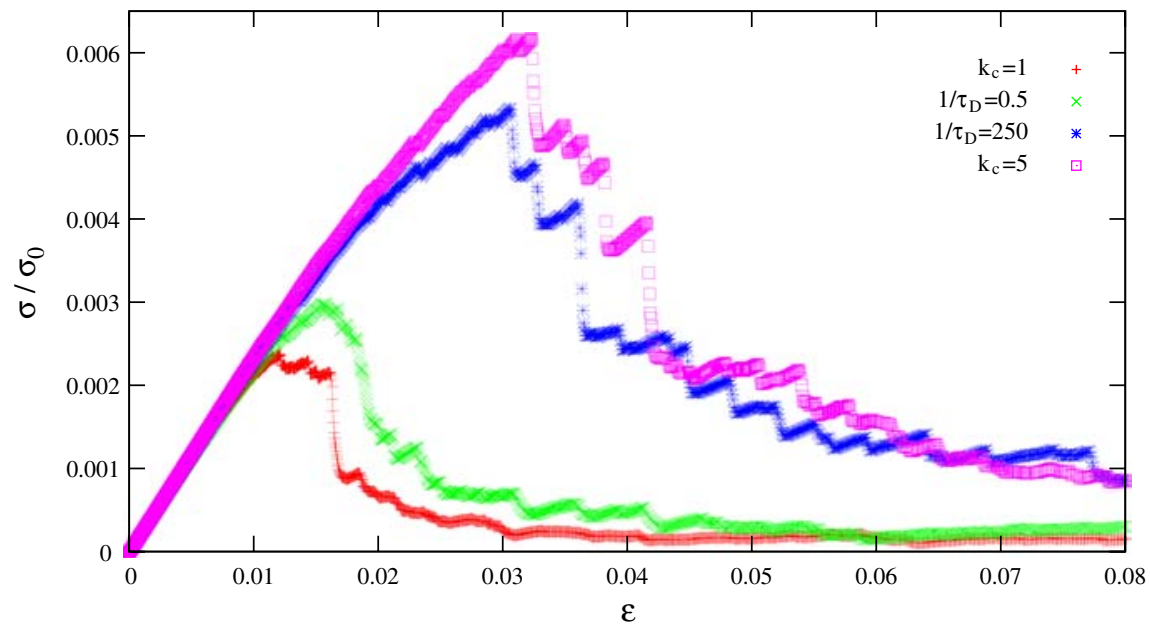


Fig. 9 Stress-strain response of samples with increasing (from bottom to top) damage detection rates $1 / \tau_{\mathrm{D}}$, as given in the inset. The curve with the highest peak stress had approximately 2,500 healing events during the compression up to strain 0.05

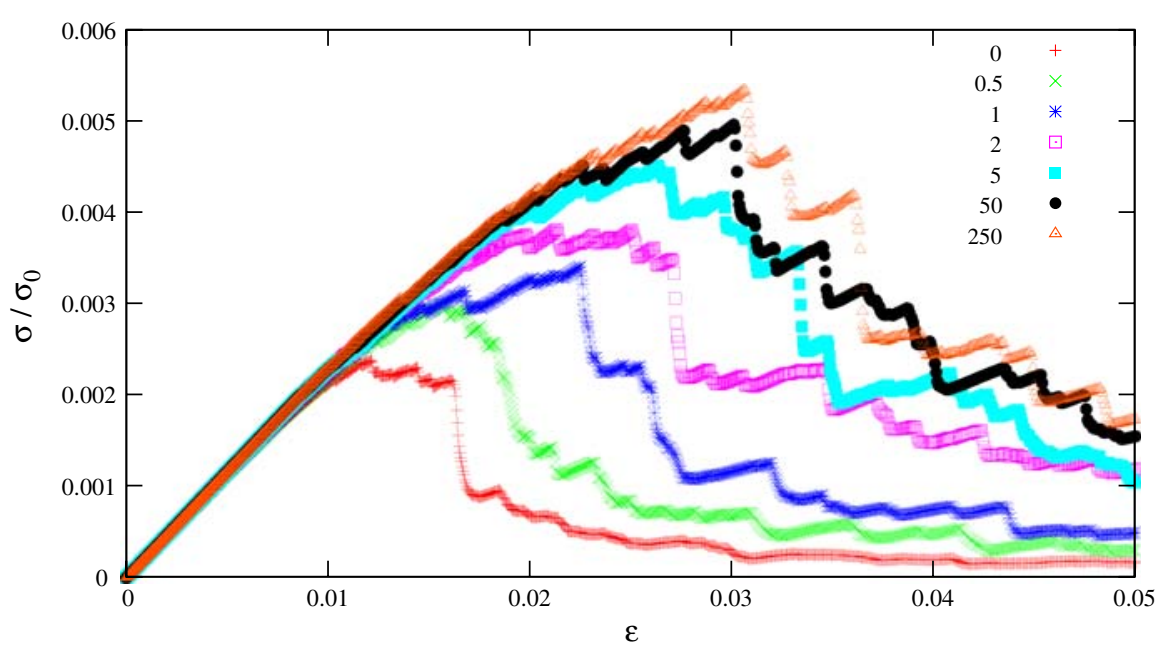

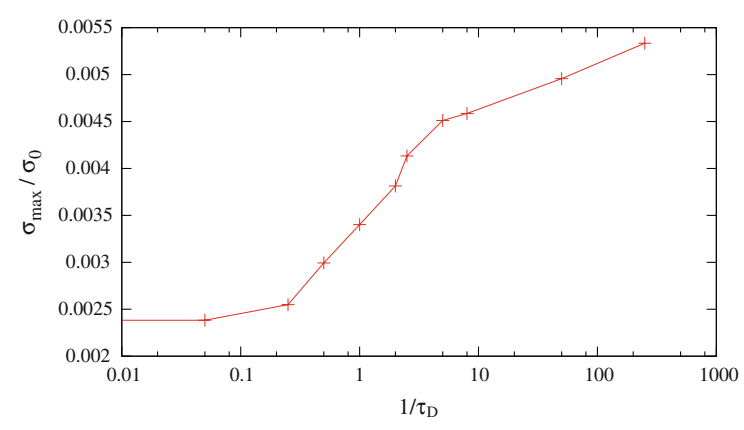

Fig. 10 Dimensionless maximal stress as function of the damage detection rate $1 / \tau_{\mathrm{D}}$

For increasing damage detection rate, both the maximum stress, see Fig. 10, and the strain where the maximum stress is reached increase (strain data not shown-they fluctuate much stronger than the peakstress). During the compression interval shown in Fig. 9 the curve with the highest peak stress (which is the one with the highest damage detection rate) was subjected to approximately 2,500 damage detection events, whereas the curve with the lowest peak stress is without self-healing.

Note that while there are small stress-drops before the peak-stress, the post-peak behavior shows much stronger rapid (brittle) stress drops and fluctuations as visible from the large separation of points in the rapid stress-drop branches. While some samples reach a clear maximum stress, in some cases (e.g., $1 / \tau_{\mathrm{D}}=2$ ) the sample can sustain a high stress over a large strain range of about 0.01 . The long "failure periods" (before the big

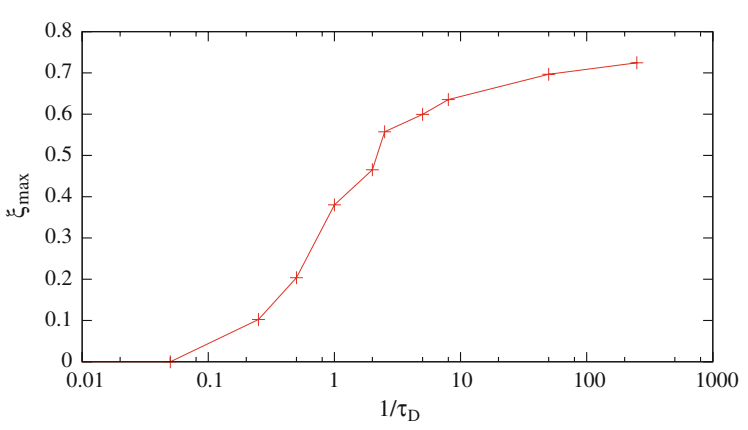

Fig. 11 Fraction $\xi_{\max }$ of healed contacts as function of the damage detection rate $1 / \tau_{\mathrm{D}}$

stress drops) sometimes resemble a plateau, sometimes they show some linear increase of stress for some short period.

An increased material strength is observed for damage detection rates greater than $1 / \tau_{\mathrm{D}} \approx 0.1$. For higher damage detection rates healing becomes more and more effective, see Fig. 10.

Comparing the peak strength to the fraction of healed contacts at peak-strength $\xi_{\max }$, see Fig. 11, reveals a similar behavior. For larger rates the strength as well as the fraction of healed contacts increase less strongly than for intermediate rates.

Overall, the strength achieved with this method-in the range of parameters examined-stays below the strength found for the "full" or "pre-emptive" or "global" healing. This leads to the conclusion that even though very large rates lead to the strongest material, a considerable effect can be achieved with a rather small damage detection rate already. This is good news for 
Fig. 12 Stress-strain response of samples with different self-healing parameters $k_{\mathrm{SH}} / k_{c}$, as given in the inset. Note that the curve with highest stress represents a sample with a self-healing adhesion parameter of (only) $k_{\mathrm{SH}} / k_{c}=50$

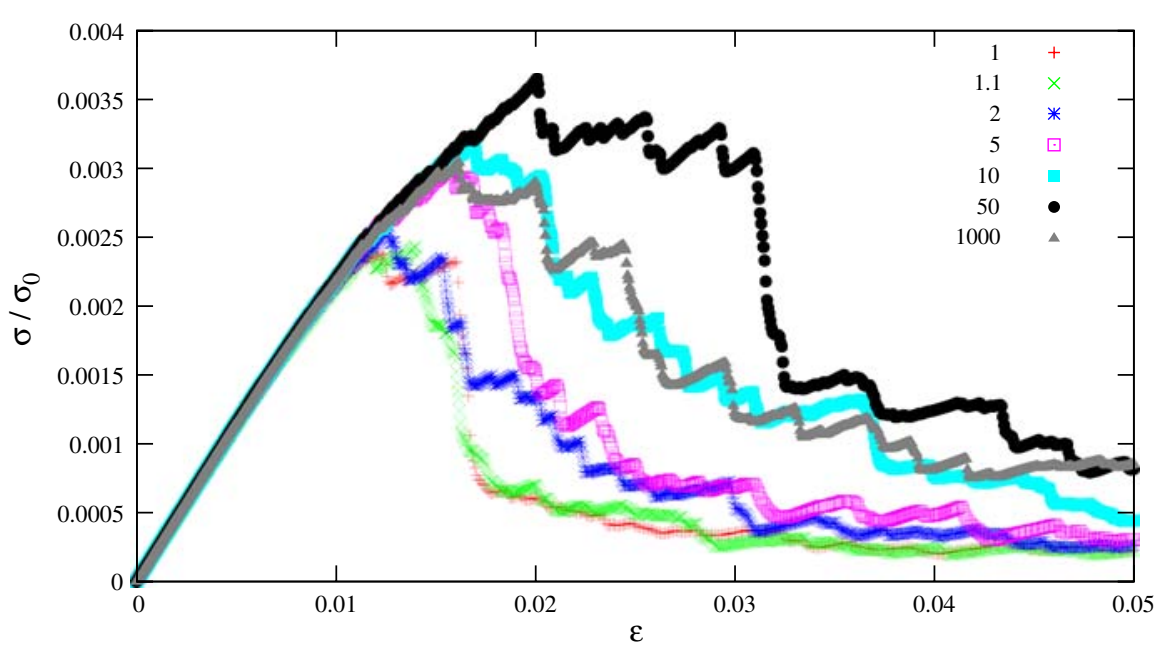

experiments as it means that the capsules containing the healing agent need not brake immediately when subjected to stress.

\subsection{Variation of adhesive force (healing intensity)}

In Fig. 12 we compare the stress-strain response for different self-healing adhesion parameters $k_{\mathrm{SH}} / k_{c}$ from 1 to $10^{3}$ but fixed the damage detection rate $1 / \tau_{\mathrm{D}}=1 / 2$. Note again the strong variation in the shape of the critical flow regime. With increasing $k_{\mathrm{SH}} / k_{c}$ the strength of the material, i.e., the maximum stress, increaseswithin the rather strong variations from one sample to the other. Interestingly, in this data-set, the largest stress is reached for $k_{\mathrm{SH}} / k_{c}=50$, and not for $k_{\mathrm{SH}} / k_{c}=10^{3}$.

After carrying out many more simulations with different healing parameters $k_{\mathrm{SH}}$, this interesting behavior can be understood, see Fig. 13. The material strength gained by self-healing saturates as the healing intensity $I_{\mathrm{SH}}$ saturates for large $k_{\mathrm{SH}} / k_{c}\left(I_{\mathrm{SH}} \rightarrow 6\right.$ for $k_{\mathrm{SH}} / k_{c} \rightarrow$ $\infty)$. It is therefore instructive to plot the maximum stress sustained not only as a function of $k_{\mathrm{SH}} / k_{c}$ (top graph of Fig. 13), but also as a function of the healing intensity (bottom graph of Fig. 13): for healing intensities $I_{\mathrm{SH}}$ up to approximately 5 (equivalent to $k_{\mathrm{SH}} / k_{c} \approx 25$ in our case) the material strength increases approximately linearly with the healing intensity. However, for larger values of $I_{\mathrm{SH}}>5\left(k_{\mathrm{SH}} / k_{c}>25\right)$ we see strong fluctuations.

We relate these strong fluctuations for $k_{\mathrm{SH}} / k_{c}>25$ $\left(I_{\mathrm{SH}}>5\right)$ to the fact that the adhesive branch in the force-displacement law, see Fig. 1, becomes extremely
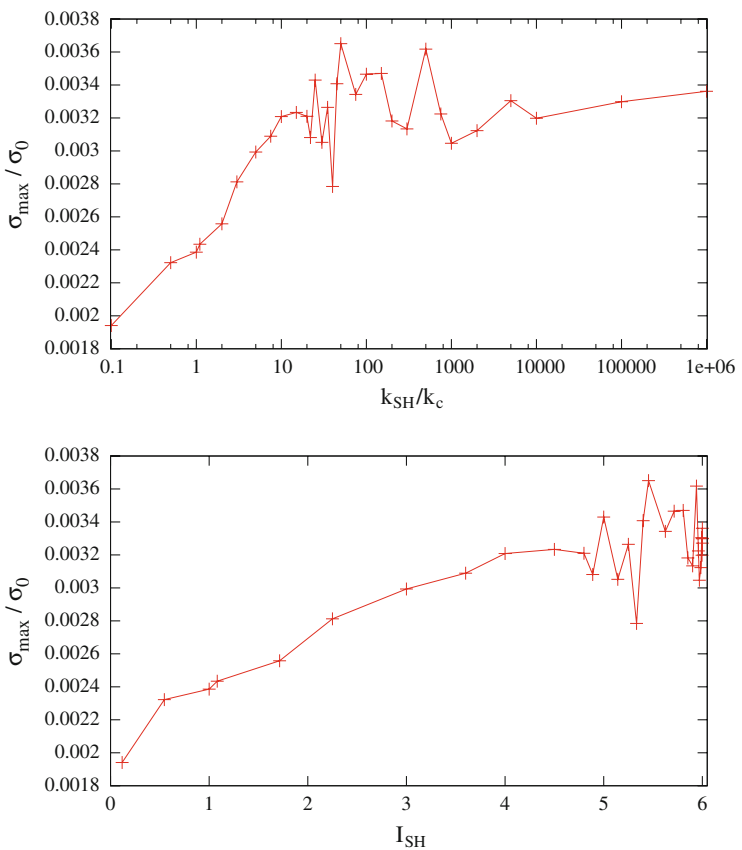

Fig. 13 (Top) Maximal stress from simulations as a function of the self-healing adhesion parameter $k_{\mathrm{SH}} / k_{c}$. (Bottom) Same stress as a function of the healing intensity, see Eq. 5.3

steep: very large $k_{\mathrm{SH}}$ (or $k_{c}$ ) values lead to a steep, almost infinite slope, while virtually not changing the maximum adhesive force. The steep slope of the model means that a contact that hits the tensile instable branch will "immediately" break (brittle) since a small tensile strain leads to an enormous drop in tensile force. This leads to strong fluctuations because the model does 


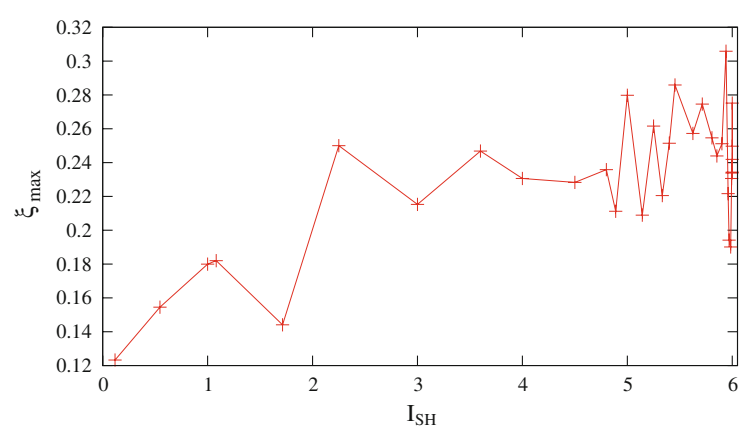

Fig. 14 Fraction of healed contacts as a function of the healing intensity $I_{\mathrm{SH}}$, see Eq. 5.3

not allow contacts to recover after they have opened $(\delta<0)$. Strong loading would be necessary to close the open contacts so that they can be healed. For smaller $k_{c}$, a small tensile strain only leads to a small drop of the tensile force and thus is less critical.

A comparison with the fraction of healed contacts as a function of the healing intensity $I_{\mathrm{SH}}$, see Fig. 14 , reveals a rather weak, discontinuous dependence of the fraction of healed contacts on the intensity of healing. There is a clearly visible trend but within large fluctuations.

\section{Summary and conclusions}

We have presented a model for self-healing in particulate materials based on a recently proposed piecewise linear contact model for elasto-plastic, adhesive, viscous, frictional particle-particle interactions. The contact model includes a memory variable, i.e., the contact laws are history dependent. The proposed self-healing model admits to set the damage detection sensitivity and rate as well as the strength of the healed contacts. It could therefore be a reasonable model for a material including a healing agent in, e.g., capsules. The model does not allow for volume change, or to close opened micro-cracks, as foaming or bubbling self-healing mechanisms could.

Uni-axial unconfined compression has been applied to isotropically pressure sintered samples and the stress response has been studied in the elastic regime, at the onset of, and during failure, as well as in the softening regime. For fast deformation the material behavior is ductile-like due to viscous, velocity dependent forces, whereas for quasi-static deformation it resembles a brittle material behavior with sharp, rapid drops in stress during failure and softening.

We found that the elastic regime can be extended using self-healing techniques. For a fixed damage sensitivity and a fixed damage detection rate, damage is detected and healed by increasing the adhesive force instantaneously if and when the healing criteria (specified in the main text) are fulfilled. The compression tests of self-healing material samples are compared to two extreme reference cases: one without healing and one with "full" or "pre-emptive" global healing, where healing has been applied to all contacts prior to the compression test. The model parameters were studied systematically, most prominently by examining the stress-strain response of the samples and additionally by monitoring the fraction of healed contacts.

The material strength (after healing) increases with (1) the damage detection sensitivity, (2) the damage detection rate, and (3) the healing intensity (which itself is a monotonically increasing function of the adhesive strength of the healed contacts).

(1) A low damage detection sensitivity leads to unsatisfactory self-healing since too few contacts will be healed. On the other hand, a very high damage detection sensitivity results in healing of contacts that are not critical. Healed contacts, within the framework of our model, once healed cannot be healed again when they become critical again in the future. This mimics, e.g., a one-time healing agent which is common in experiments where, e.g., capsules are used.

(2) The damage detection rate allows to set a delay or relaxation time between damage detection events. Too small a damage detection rate does not allow for (enough) healing to take place as many critical contacts are missed whereas for very large damage detection rates the material strength should saturate once the detection rate has reached the smallest physical time scale. The damage detection rate, together with the damage detection sensitivity, allows to simulate various types of responses to damage, e.g., more brittle or softer capsules containing the healing agent and/or different response time scales of the healing agent.

(3) The healing intensity is a non-linear function of the adhesion parameter $k_{\mathrm{SH}}$. It saturates for large $k_{\mathrm{SH}}$ and sets the strength of the healed contacts. With increasing healing intensity the material can sus- 
tain larger stresses and fails at higher strains. As a function of $k_{\mathrm{SH}}$ the material strength shows strong fluctuations for large $k_{\mathrm{SH}}$, i.e., in the regime where the healing intensity is almost constant. These fluctuations are due to local contact instabilities. For very large healing intensities, i.e., for very large adhesive strength of the healed contacts, a contact rapidly fails when the tensile limit is reached, resembling a local, brittle failure at the contact level. Thus, moderate values for the adhesive constant after healing, $k_{\mathrm{SH}}$, lead to the "best" healing results. The fraction of healed contacts increases a little with the healing intensity, which corresponds to the final strength of the (solidified) healing agent, i.e., the strength of the final bonding.

To sum up, our model is consistent with, e.g., materials containing healing-agents in capsules. Once a capsule is broken and local healing has taken place, there cannot be any further healing at the same position. Different types of capsules and capsule-matrix interface can be modeled by different damage detection sensitivities and damage detection rates. Different bonding strengths of the healing agent can be modeled by adjusting the self-healing adhesive strength $k_{\mathrm{SH}}$.

The quantitative tuning of the DEM model to real experimental data remains a challenge for future research. The results presented here have units that are not (yet) supposed to mimic a real material. Some tuning can be done by rescaling, but a real fine-adjustment will require a comparison with appropriate experimental data.

The model can also be extended to include an additional time scale on which-after damage is detected and healing is initiated - the strength slowly increases to mimic the "bonding" or "hardening" of the healing agent. Work along this line is in progress. Another way to extend this work could be to allow for repeated healing through a cascade of healing with ever increasing adhesive contact force (at the same position). The final challenge remains to observe a healing result that is superior to the (much simpler) global, pre-emptive healing of all contacts.

Acknowledgements The authors wish to thank Akke Suiker, Orion Mouraille, and Christine Herbst for useful discussions. This study was made possible by the Delft Center for Materials Self-Healing program, and supported by the research institute IMPACT of the University of Twente, and the Stitching voor Fundamenteel Onderzoek der Materie (FOM), financially sup- ported by the Nederlandse Organisatie voor Wetenschappelijk Onderzoek (NWO), through the Granular Matter program.

Open Access This article is distributed under the terms of the Creative Commons Attribution Noncommercial License which permits any noncommercial use, distribution, and reproduction in any medium, provided the original author(s) and source are credited.

\section{References}

Abrams DA (1918) Design of concrete mixtures Bulletin I. Structural Materials Research Laboratory, Lewis Institute, Chicago, Illinois 309-330

Agnolin I, Jenkins JT, Ragione LL (2006) A continuum theory for a random array of identical, elastic, frictional disks. Mech Mater 38(8-10):687-701

Allen MP, Tildesley DJ (1987) Computer simulation of liquids. Oxford University Press, Oxford

Balazs AC (2007) Modeling self-healing materials. Mater Today 10(9):18-23

Barbero E, Greco F, Lonetti P (2005) Continuum damagehealing mechanics with application to self-healing composites. Int J Damage Mech 14(1):51-81

Bashir YM, Goddard JD (1991) A novel simulation method for the quasi-static mechanics of granular assemblages. J Rheol 35(5):849-885

Behringer RP (1993) The dynamics of flowing sand. Nonlinear Sci Today 3:1-15

Behringer RP, Baxter GW (1994) Pattern formation and complexity in granular flow. In: Mehta A (ed) Granular matter. 85

Brandeis F (1937) Autogenous healing of concrete. Beton und Eisen 36:12

Brown E, Sottos N (2000) Performance of embedded microspheres for self-healing polymer composites, preprint http://www.autonomic.uiuc.edu/brown_files/EricBrownSE M2000.pdf

Brown EN, Kessler MR, Sottos NR (2003a) In situ poly(ureaformaldehyde) microencapsulation of dicyclopentadiene. J Microencapsul 20:719-730

Brown EN, Moore JS, White SR, Sottos NR (2003b) Fracture and fatigue behavior of a self-healing polymer. Mat Res Soc Symp Proc 735: C11.22.1

Brown EN, White SR, Sottos NR (2005) Retardation and repair of fatigue cracks in a microcapsule toughened epoxy composite part II: in situ self-healing. Compos Sci Technol 65:2474-2480

Burton DS, Goa X, Brinson LC (2006) Finite element simulation of a self-healing shape memory alloy composite. Mech Mater 38(5-6):525-537

Castellanos A (2005) The relationship between attractive interparticle forces and bulk behavior in dry and uncharged fine powders. Adv Phys 54(4):263-376

Cordier P, Tournilhac F, Soulié-Ziakovic C, Leibler L (2008) Self-healing and thermoreversible rubber from supramolecular assembly. Nature 451:977-980

Cundall PA, Strack ODL (1979) A discrete numerical model for granular assemblies. Géotechnique 29(1):47-65 
David CT, Rojo RG, Herrmann HJ, Luding S (2005) Hysteresis and creep in powders and grains. In: Garcia-Rojo R, Herrmann HJ, McNamara S (eds) Powders and grains 2005. Balkema Leiden, Netherlands pp 291-294

Dry C (1994) Matrix cracking repair and filling using active and passive modes for smart timed release of chemicals from fibers into cement matrices. Smart Mater Struct 3:118-123

Dry C (1996a) Smart bridge and building materials in which cyclic motion is controlled by internally released adhesives. Proc SPIE 2719:247-254

Dry C (1996b) Procedures developed for self-repair of polymeric matrix composite materials. Compos Struct 35:263-269

Dry CM Smart-fiber-reinforced matrix composites, U.S. Patent $5,803,963$

Feng W, Patel SH, Young M-Y, Zunino JLIII, Xanthos M (2007) Smart polymeric coatings-recent advances. Adv Polym Technol 26(1):1-13

Goldhirsch I, Zanetti G (1993) Clustering instability in dissipative gases. Phys Rev Lett 70(11):1619-1622

Guo Y, Guo W (2006) Self-healing properties of flaws in nanoscale materials: Effects of soft and hard molecular dynamics simulations and boundaries studied using a continuum mechanical model. Phys Rev B 73:085411

Hearn N (1998) Self-sealing, autogenous healing and continued hydration: what is the difference?. Mater Struct/Materiaux et Constructions 31:563-567

Herbst O, Huthmann M, Zippelius A (2000) Dynamics of inelastically colliding spheres with Coulomb friction: relaxation of translational and rotational energy. Granul Matter 2(4):211-219

Herbst O, Cafiero R, Zippelius A, Herrmann HJ, Luding $\mathrm{S}$ (2005) A driven two-dimensional granular gas with Coulomb friction. Phys Fluids 17:107102

Herrmann HJ, Hovi J-P, Luding S (eds) (1998) Physics of dry granular media-NATO ASI Series E 350. Kluwer Academic Publishers, Dordrecht

Jaeger HM, Nagel SR (1992) Physics of the granular state. Science 255:1523

Jaeger HM, Liu C, Nagel SR (1989) Relaxation at the angle of repose. Phys Rev Lett 62(1):40-43

Jaeger HM, Liu C, Nagel SR, Witten TA (1990) Friction in granular flows. Europhys Lett 11(7):619-624

Janssen HA (1895) Versuche über Getreidedruck in Silozellen. Zeitschr d Vereines deutscher Ingenieure 39(35):10451049

Kafui KD, Thornton C (2000) Numerical simulations of impact breakage of spherical crystalline agglomerate. Powder Technol 109:113-132

Kersey FR, Loveless DM, Craig SL (2007) A hybrid polymer gel with controlled rates of cross-link rupture and self-repair. J Roy Soc Interface 4:373-380

Kessler MR (2007) Self-healing: a new paradigm in materials design, Proc I MECH E Part G J Aerosp Eng 221(4):479_ 495(17)

Kessler MR, Sottos NR, White SR (2003) Self-healing structural composite materials. Compos Part A Appl Sci Manuf 34(8):743-753

Lätzel M, Luding S, Herrmann HJ, Howell DW, Behringer RP (2003) Comparing simulation and experiment of a $2 d$ granular couette shear device. Eur Phys J E 11(4):325333
Li VC, Lim YM, Chan Y-W (1998) Feasibility study of a passive smart self-healing cementitious composite. Compos Part B 29:819-827

Luding S (2005a) Shear flow modeling of cohesive and frictional fine powder. Powder Technol 158:45-50

Luding S (2005b) Anisotropy in cohesive, frictional granular media. J Phys Condens Matter 17:S2623-S2640

Luding S (2008) Cohesive frictional powders: contact models for tension. Granul Matter 10:235-246

Luding S, Herrmann HJ (2001) Micro-macro transition for cohesive granular media. In: Diebels S (Ed) Bericht Nr. II-7, Inst für Mechanik, Universität Stuttgart

Luding S, Suiker ASJ (2008) Self-healing of damaged particulate materials through sintering. Philos Mag 88(28-29):34453457

Luding S, Clément E, Blumen A, Rajchenbach J, Duran J (1994a) Studies of columns of beads under external vibrations. Phys Rev E 49(2):1634

Luding S, Clément E, Blumen A, Rajchenbach J, Duran J (1994b) Anomalous energy dissipation in molecular dynamics simulations of grains: The "detachment effect". Phys Rev E 50:4113

Luding S, Manetsberger K, Muellers J (2005) A discrete model for long time sintering. J Mech Phys Solids 53(2):455-491

Maiti S, Shankar C, Geubelle PH, Kieffer J (2006) Continuum and molecular-level modeling of fatigue crack retardation in self-healing polymers. J Eng Mater Technol 128(4):595602

Mauldin TC, Rule JD, Sottos NR, White SR, Moore JS (2007) Self-healing kinetics and the stereoisomers of dicyclopentadiene. J Roy Soc Interface 4:389-393

Peizhen H, Zhonghua L, Jun S (2000) Finite element analysis on evolution process for damage microcrack healing. Acta Mechanica Sinica 16(3):254-263

Pöschel T, Schwager T (2005) Computational granular dynamics. Springer, Berlin

Priman V, Dementsov A, Sokolov I (2007) Modeling of selfhealing polymer composites reinforced with nanoporous glass fibers. J Comp Theor Nanosci 4:190-193

Rapaport DC (1995) The art of molecular dynamics simulation. Cambridge University Press, Cambridge

Santos A (2008) Does the Chapman-Enskog expansion for sheared granular gases converge? Phys Rev Lett 100:078003

Schwedes J (2003) Review on testers for measuring flow properties of bulk solids. Granul Matter 5(1):1-45

Sela N, Goldhirsch I (1998) Hydrodynamic equations for rapid flows of smooth inelastic spheres to Burnett order. J Fluid Mech 361:41-74

Shchukin DG, Möhwald H (2007) Self-repairing coatings containing active nanoreservoirs. SMALL 3(6):926-943

Sijbesma RP, Beijer FH, Brunsveld L, Folmer BJB, Hirschberg JHKK, Lange RFM, Lowe JKL, Meijer EW (1997) Reversible polymers formed from self-complementary monomers using quadruple hydrogen bonding. Science 278:16011604

Soroker VJ, Denson AJ (1926) Autogenous healing of concrete. Zement 25:30

Sperl M (2006) Experiments on corn pressure in silo cells. translation and comment of janssen's paper from 1895. Granul Matter 8(2):59-65 
Thornton C (2000) Numerical simulations of deviatoric shear deformation of granular media. Géotechnique 50(1):4353

Thornton C, Antony SJ (2000) Quasi-static deformation of a soft particle system. Powder Technol 109(1-3):179-191

Thornton C, Yin KK (1991) Impact of elastic spheres with and without adhesion. Powder Technol 65:153

Thornton C, Zhang L (2001) A DEM comparison of different shear testing devices. In: Kishino YPowders and grains. Balkema, Rotterdam pp 183-190

Thornton C, Yin KK, Adams MJ (1996) Numerical simulation of the impact fracture and fragmentation of agglomerates. J Phys D Appl Phys 29:424-435

Tomas J (2004) Fundamentals of cohesive powder consolidation and flow. Granul Matter 6(2/3):75-86

Trask RS, Williams HR, Bond IP (2007a) Self-healing polymer composites: mimicking nature to enhance performance. Bioinsp Biomim 2:1-9

Trask RS, Williams GJ, Bond IP (2007b) Bioinspired selfhealing of advanced composite structures using hollow glass fibres. J Roy Soc Interface 4:363-371

Turner L The autogenous healing of cement and concrete-its relation to vibrated concrete and cracked concrete, Proc. Int Assoc. Testing Materials. Testing Materials, London van der Zwaag S (ed) (2007) Self-Healing Materials. Springer Vermeer PA, Diebels S, Ehlers W, Herrmann HJ, Luding S, Ramm E (eds) (2001) Continuous and discontinuous modelling of cohesive frictional materials. Springer, Berlin. lecture Notes in Physics 568

Vermeer PA, Ehlers W, Herrmann HJ, Ramm E (eds) (2004) Modeling of cohesive-frictional materials. Balkema, Leiden, Netherlands (ISBN 041536023 4)

Wagner EF (1974) Autogenous healing of cracks in cementmortar linings for grey-iron and ductile-iron watered pipes. J Am Water Works Assoc 66:358-360

Williams GJ, Trask RS, Bond IP (2007) A self-healing carbon fibre reinforced polymer for aerospace applications. Compos Part A Appl Sci Manuf 38(6):1525-1532

White SR, Sottos NR, Geubelle PH, Moore JS, Kessler MR, Sriram SR, Brown EN, Viswanathan S (2001) Autonomic healing of polymer composites. Nature 409:794-797

White SR, Maiti S, Jones AS, Brown EN, Sottos NR, Geubelle PH (2004) Fatigue of self-healing polymers: multiscale analysis and experiments, preprint. http://www.icf11. com/proceeding/EXTENDED/5414.pdf

White SR, Sottos NR, Geubelle PH, Moore JS, Sriram SR, Kessler MR, Brown EN Multifunctional autonomically healing composite material, U.S. Patent 6,518,330 